\title{
Clusterization and Deformation in Heavy Nuclei
}

\author{
A. Algora*, J. Cseh ${ }^{*}$, J. Darai ${ }^{\dagger}$, P. O. Hess ${ }^{* *}$, N. V. Antonenko ${ }^{\ddagger}$, R. V. Jolos ${ }^{\ddagger}$ \\ and W. Scheid \\ ${ }^{*}$ Institute of Nuclear Research, Debrecen, Hungary \\ ${ }^{\dagger}$ Institute of Experimental Physics, University of Debrecen, Debrecen, Hungary \\ ${ }^{* *}$ Instituto de Ciencias Nucleares, UNAM, Mexico \\ ${ }^{\ddagger}$ Institut für Theoretische Physik der Justus-Liebig-Universität, Giessen, Germany
}

\begin{abstract}
The deformation-dependence of clusterization in heavy nuclei is investigated. In particular, allowed and forbidden cluster-configurations are determined for the ground, superdeformed, and hyperdeformed states of some nuclei, based on a microscopic (effective $S U(3)$ ) selection rule. The stability of the different cluster configurations from the viewpoint of the binding energy and the dinuclear system model (DNS) is also investigated.
\end{abstract}

Keywords: clusterization and deformation in heavy nuclei, energetic stability, Pauli-principle PACS: 21.60.Fw, 21.60.Gx.

When studying the relation between the quadrupole deformation and clusterization of light nuclei the energetic aspect of the problem can be handled in terms of empirical rules or phenomenologic models, while the effect of the Pauli-exclusion principle can be taken into account via a selection rule based on the $U(3)$ symmetry [1]. For heavy nuclei the treatment of the energetics is applicable in the same way, however, the $U(3)$ symmetry is not valid due to the importance of the spin-orbit and other symmetry-breaking interactions. Nevertheless, a more general symmetry, called effective or quasidynamical symmetry [2] can still be helpful.

The quasidynamical or effective symmetry is one of the most general symmetry concepts in quantum mechanics. It is a symmetry of the eigenvalue equation, when neither the (Hamiltonian) operator is symmetric (scalar), nor its eigenvectors (transform according to some irreducible representation) [3]. Yet it may act, and have important physical consequences. The mathematical reason for this surprising situation is provided by the embedded representation [2,4]. An embedded representation is spanned by a set of states that are linear combinations of basis states from similar but inequivalent irreducible representations (irreps).

In particular for the nuclear $U(3)$ case the difference between the usual dynamical symmetry and the quasidynamical symmetry is as follows. In the Elliott-model [5] (and similarly in some other models) the energy-eigenvectors are $U(3)$ basis states, though the Hamiltonian is not invariant with respect to $U(3)$. This is due to the fact that the symmetry-breaking interaction can be expressed in terms of the invariant operator of $S O(3)$, being a subgroup of $U(3)$ :

$$
\begin{aligned}
& U(3) \supset S U(3) \supset S O(3) \\
& {\left[n_{1} n_{2} n_{3}\right](\lambda \mu) K J .}
\end{aligned}
$$

(Here we have indicated also the quantum numbers that characterize the irreducible 
representations.) Thus the Hamiltonian is obtained in terms of the invariant operators of a single chain of nested subgroups. In such a case the degeneracy, which would characterize the exact $U(3)$ symmetry, is split but the different irreps of $U(3)$ are not mixed.

In case of the quasidynamical or effective symmetry the breaking is stronger, basis states of different $S U(3)$ irreps are mixed with each other:

$$
\psi_{\alpha K J M}=\sum_{\xi \lambda \mu} C_{\alpha \xi \lambda \mu K} \phi \xi \lambda \mu K J M
$$

where $\phi_{\xi \lambda \mu K J M}$ is a basis vector for an $S U(3)$ irrep, and $\xi$ stands for all the quantum numbers not belonging to group-chain (1) [6]. Please, note that the $C_{\alpha \xi \lambda \mu K}$ coefficients of the linear combination are independent of $J M$, i.e. within a band the contribution of different $S U(3)$ basis states are the same. Due to this feature these bands are called soft $S U(3)$ bands, contrary to the rigid $S U(3)$ bands, defined by fixed and unique $(\lambda, \mu)$ values. If calculating the matrix elements of the $S U(3)$ generators between these states the result may approximate the matrix elements of an exact representation. In such a case we speak about an approximate embedded representation, and related to it about an approximate quasidynamical or effective symmetry.

From the physical point of view the necessary condition for the survival of the effective $S U(3)$ symmetry is that the rotational frequencies are adiabatic relative to the complementary intrinsic degrees of freedom (c.f. $J M$-independent coefficients).

The embedded representation and the related quasidynamical symmetry explains, why the $S U$ (3) model (and the rotational band structure) survives well beyond the limit one would expect, based on the effects of the symmetry-breaking interactions.

The effective $U(3)$ symmetry provides us with effective (or average) $U(3)$ quantum numbers, and based on that a selection rule can be formulated. In [6] the procedure for determining the effective $S U(3)$ quantum numbers were determined for large prolate deformation, but it can be generalized for oblate shape and for small deformations as well [7]. Based on the effective $U(3)$ quantum numbers one can apply the $U(3)$ selection rule, as described in [1].

In what follows, we present the application of our method to two heavy systems $\left({ }^{252} \mathrm{Cf},{ }^{232} \mathrm{Th}\right)$. The ${ }^{252} \mathrm{Cf}$ nucleus attracted much attention due to recent experiments published in [8]. The ${ }^{232} \mathrm{Th}$ case is of interest because theoretical calculations [9] have shown that the structure of the third minimum in this nucleus resembles a binuclear configuration composed of a spherical heavy fragment around ${ }^{132} \mathrm{Sn}$ and a well deformed lighter fragment around ${ }^{100} \mathrm{Zr}$. Furthermore, experimental activity is going on in this region [10] in order to check the relation between the hyperdeformed state and clusterizations.

We have studied all possible binary clusterizations of ${ }_{98}^{252} \mathrm{Cf} \rightarrow{ }_{Z}^{A} \mathrm{X}+{ }_{98-Z}^{252-A} \mathrm{Y}$ (308 fission channels with $8 \leq Z \leq 90$ ). The starting point is always the deformation of the nucleus (parent or cluster). Then we fill in the Nilsson orbitals from below at that deformation value and determine the effective $(\lambda, \mu)$ using the relations of $[6,7]$. The parameters for the Nilsson Hamiltonian where taken from [11]. Using the information on the occupation of the main oscillator shells it is also possible to apply the Harvey prescription [12] for the determination of the minimum number of quanta of the relative motion. 

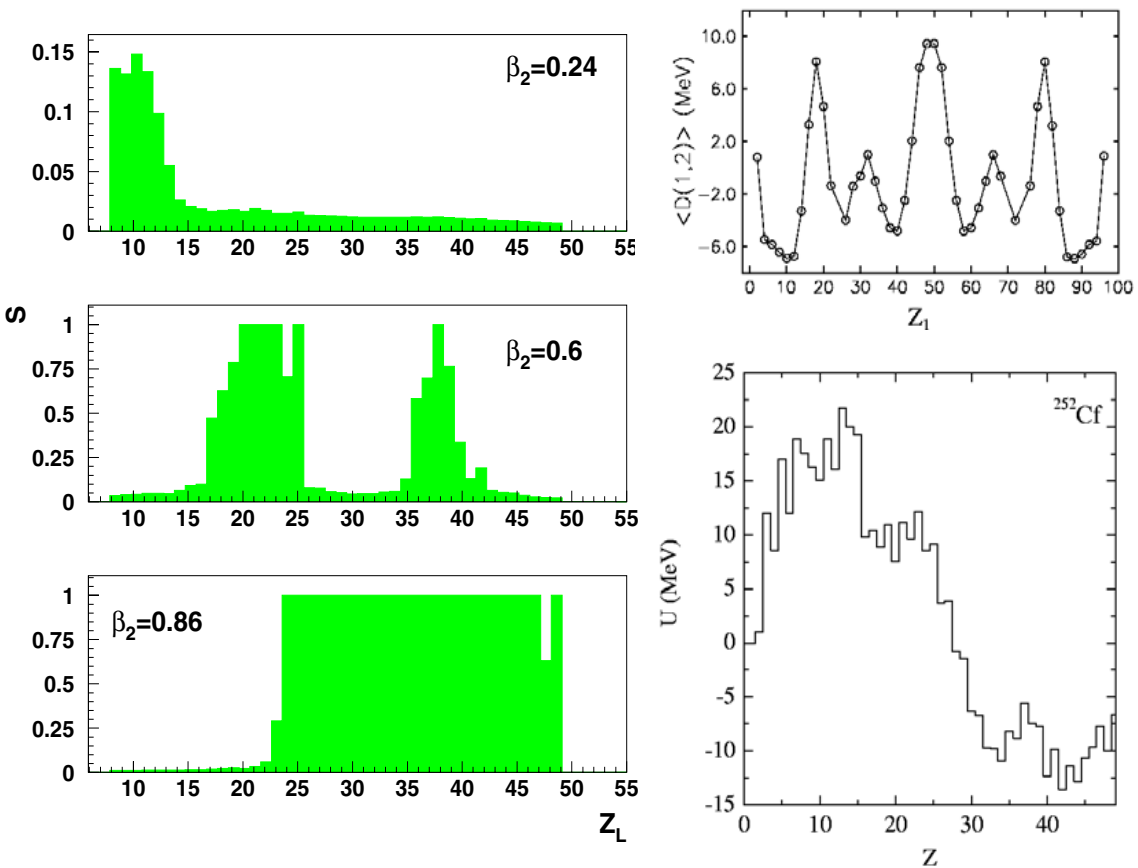

FIGURE 1. Left panel: reciprocal forbiddenness $S$ versus the $Z_{\text {light }}$ of the studied binary cluster configurations for the ${ }^{252} \mathrm{Cf}$ case. (For the definitions of the $\mathrm{S}, \mathrm{D}(1,2)$, and $\mathrm{U}$ see [1].) The values of $\mathrm{S}$ correspond to mean values over channels that have the same $Z_{\text {light }}$ and different $A_{\text {light }}$. Right panel (upper part): energetic stability of binary clusterizations in ${ }^{252} \mathrm{Cf}$ based on binding energies. Right panel (lower part): results from the DNS model calculations for ${ }^{252} \mathrm{Cf}[15]$.

Assuming ground state deformations for the parent and daughter nuclei [13], all studied cluster configurations in question turn out to be forbidden (Fig. 1, left panel, upper part) [14]. A clear tendency towards cluster radioactivity (or very asymmetric fission) can be inferred from this figure. Within this framework we have also addressed the question if there are allowed clusterizations in case when we change the deformation of the parent nucleus to superdeformation $\left(\beta_{2} \sim 0.6\right)$ or hyperdeformation $\left(\beta_{2} \sim 0.86\right)$. The obtained results are presented in the lower parts of Fig. 1 (left panel, lower parts). As in the earlier calculations the clusters are considered to have ground state deformations. It is interesting to see that in these cases there are allowed clusterizations as well. Assuming a superdeformed ${ }^{252} \mathrm{Cf}$ the regions of allowed clusterizations correspond mainly to two particular regions in which: a) both clusters have large prolate quadrupole deformation (region with $\mathrm{Z}_{\text {light }} \sim 36$ ), b) one cluster with prolate quadrupole deformation and the other with oblate deformation (region with $\mathrm{Z}_{\text {light }} \sim 22$ ). For the hyperdeformed ${ }^{252} \mathrm{Cf}$ case more channels are open, and from Fig. 1 (left panel, lower part) a clear tendency to symmetric clusterization can be inferred. 
TABLE 1. Some selected ternary clusterizations of ${ }^{252} \mathrm{Cf}$ (for details see the text). $\mathrm{D}(1,2,3)$ shows the energetic stability based on the binding energies.

\begin{tabular}{|c|c|c|c|c|}
\hline \multirow{2}{*}{$C_{1}+C_{2}+C_{3}$} & \multicolumn{3}{|c|}{$\mathrm{S}$} & $\mathrm{D}(1,2,3)$ \\
\cline { 2 - 4 } & $\mathrm{ND}$ & $\mathrm{SD}$ & $\mathrm{HD}$ & \\
\hline${ }^{208} \mathrm{~Pb}+{ }^{40} \mathrm{Si}+{ }^{4} \mathrm{He}$ & 0.0412 & 1.0000 & 0.0186 & 24.78 \\
${ }^{208} \mathrm{~Pb}+{ }^{22} \mathrm{O}+{ }^{22} \mathrm{O}$ & 0.0343 & 1.0000 & 0.0222 & 24.66 \\
\hline${ }^{132} \mathrm{Sn}+{ }^{70} \mathrm{Ni}+{ }^{50} \mathrm{Ca}$ & 0.0099 & 0.0178 & 0.0343 & 23.80 \\
\hline${ }^{78} \mathrm{Ni}+{ }^{126} \mathrm{Sn}+{ }^{48} \mathrm{Ca}$ & 0.0100 & 0.0178 & 0.0343 & 23.78 \\
${ }^{78} \mathrm{Ni}+{ }^{90} \mathrm{Kr}+{ }^{84} \mathrm{Se}$ & 0.0099 & 0.0174 & 0.0329 & 20.65 \\
\hline${ }^{48} \mathrm{Ca}+{ }^{132} \mathrm{Sn}+{ }^{72} \mathrm{Ni}$ & 0.0102 & 0.0186 & 0.0374 & 24.77 \\
\hline
\end{tabular}

We have also studied the criterion of maximum stability for comparison. The results of our study are presented in Fig. 1 (right panel, upper part). It is clear from this figure that the preferred clusterizations of the ground state of ${ }^{252} \mathrm{Cf}$ concentrate mainly in three regions around $Z=2, Z \sim 18$ and $Z \sim 50$. This result agrees only with our $U(3)$ procedure for the $Z=2$ case (the $\alpha$ clusterization is allowed in the $U(3)$ framework). The other two regions are not favored in the $U(3)$ approach if we consider ${ }^{252} \mathrm{Cf}$ in its ground state deformation (Fig. 1, left panel, upper part), but $Z \sim 18$ is preferred if we assume ${ }^{252} \mathrm{Cf}$ in a superdeformed state and the $Z \sim 50$ is allowed in the case of the hyperdeformed state. Also in Fig. 1 (right panel, lower part) the results of the DNS calculations are presented. The results of this calculation show that rather symmetric clusterizations are preferred in this approach, which again only agrees with our $U(3)$ prediction for the HD state case.

We have started to study possible ternary clusterizations of ${ }^{252} \mathrm{Cf}$. Table 1 shows some preliminary results in the case when one cluster is fixed as a double magic nucleus, and the other two clusters are selected using the division dictated by the criterion of maximum stability applied to the residual nucleus (once the double magic nucleus is subtracted from the ${ }^{252} \mathrm{Cf}$ ). In this particular case our results show that only clusterizations related to ${ }^{208} \mathrm{~Pb}$ double magic nucleus are allowed, and only when ${ }^{252} \mathrm{Cf}$ is assumed in a SD state. A more systematic study is underway [16].

One question of particular interest that can be addressed in the $U(3)$ framework is if the ${ }^{232} \mathrm{Th} \rightarrow{ }^{100} \mathrm{Zr}+{ }^{132} \mathrm{Sn}$ clusterization is an allowed one. This clusterization is located at the maximum of the mass distribution of the fission of ${ }^{232} \mathrm{Th}$. Another reason for studying this particular clusterization is that in [9] it was shown that the structure of the third minimum in ${ }^{232} \mathrm{Th}$ corresponds to a bi-nuclear configuration involving a spherical heavy fragment around ${ }^{132} \mathrm{Sn}$ and a well deformed lighter fragment around ${ }^{100} \mathrm{Zr}$

In a first step we have studied if this clusterization is allowed assuming that the parent nucleus and the clusters have ground state deformations $\left({ }^{232} \mathrm{Th}\left(\beta_{2} \sim 0.2\right),{ }^{100} \mathrm{Zr}\right.$ $\left.\left(\beta_{2} \sim 0.36\right),{ }^{132} \mathrm{Sn}\left(\beta_{2} \sim 0.0\right)\right)$. The $\mathrm{U}(3)$ selection rule shows that this clusterization is not allowed. The studied clusterization remains forbidden even if we assume that ${ }^{232} \mathrm{Th}$ is in a hypothetical superdeformed ( $\mathrm{SD}, \beta_{2} \sim 0.6$ ) or hyperdeformed (HD, $\beta_{2} \sim 0.86$ ) state, keeping the clusters in their ground state deformations. 
In a second step we have studied if the ${ }^{100} \mathrm{Zr}+{ }^{132} \mathrm{Sn}$ clusterization is allowed when we change the deformation of the clusters. Considering the high stability against deformation of the double magic nucleus ${ }^{132} \mathrm{Sn}$, we have changed the deformation of ${ }^{100} \mathrm{Zr}$. ${ }^{100} \mathrm{Zr}$ can be considered soft against deformation, and it is located in a region of shape coexistence [17]. Assuming ground state deformation for ${ }^{232} \mathrm{Th}$, the clusterization remains forbidden when we change the deformation of ${ }^{100} \mathrm{Zr}$. A different result is obtained when we assume that the ${ }^{232} \mathrm{Th}$ is in a SD or in a HD state. In these cases the studied clusterization is allowed when the ${ }^{100} \mathrm{Zr}$ has large oblate deformation $\left(\beta_{2} \leq\right.$ $-0.4)$.

Summarizing, in this contribution we have studied the effect of deformation in the clustering phenomena of heavy nuclei. Both the microscopic aspects of the problem (exclusion principle) and the energetics were taken into account. Our conclusions are very much in line with those found for light nuclei [1]: the energetic stability and the Pauli-principle turn out to play a complementary role, therefore, they do not necessarily result in the same preference of cluster configurations. The most likely clusterizations are those, which are preferred from both aspects.

This work was supported by the OTKA (Grant Nos. T37502, T46791), the MTACONACyT joint project and by DGAPA (IN119002). A. Algora recognizes partial support of the János Bolyai research fellowship, while J. Cseh that of the Alexander von Humboldt foundation.

\section{REFERENCES}

1. J. Cseh, contribution to this conference.

2. D. J. Rowe, Computational and Group-Theoretical Methods in Nuclear Physics, Eds: J. Escher et al., World Scientific, Singapore, 2004, p.165.

3. J. Cseh, Rev. Mex. Fis. 49 Suppl. 4. (2003) 101;

J. Cseh, Nuclear Theory22, Ed: V. Nikolaev, Heron Press, Sofia, 2003, p. 184.

4. P. Rochford, D. J. Rowe, Phys. Lett B 210 (1988) 5;

D. J. Rowe, P. Rochford, J. Repka, J. Math. Phys. 29 (1988) 572.

5. J. P. Elliot, Proc. R. Soc. A 245 (1958) 128, 562.

6. M. Jarrio, J. L. Wood, and D. J. Rowe, Nucl. Phys. A528 (1991) 409.

7. P. O. Hess, A. Algora, M. Hunyadi, and J. Cseh, Eur. Phys. J. A15 (2002) 449.

8. J. H. Hamilton et al, J. Phys. G20 (1994) L85;

A. V. Ramaya et al, Phys. Rev. Lett. 81 (1998) 947.

9. S. Cwiok, W. Nazarewicz, J. X. Saladin, W. Plociennik and A. Johnson, Phys. Lett. B 322 (1994) 304.

10. A. Krasznahorkay et al., Heavy Ion Phys. 18 (2003) 323.

11. T. Bengtsson and I. Ragnarsson, Nucl. Phys. A436 (1985) 14

12. M. Harvey, Proceedings of the 2nd Conference on Clustering Phenomena on Nuclei, College Park, 1975, USDERA Report ORO-4856-26, p. 549.

13. P. Möller, J. R. Nix, W. D. Myers and W. J. Swiatetecki, At.Nucl. Data Tables 59, 185 (1995).

14. J. Cseh, A. Algora, J. Darai, P. O. Hess, Phys. Rev. C70 (2004) 034311.

15. V.V. Volkov, Phys. Rep. 44, 93 (1978);

V. V. Deep inelastic nuclei reactions (Energoizdat, Moscow, 1982);

W. Greiner, J.Y. Park, and W. Scheid, Nuclear Molecules(World Scientific, Singapore, 1995);

G.G. Adamian et al., Phys. Rev. C67 (2003) 054303; C69 (2004) 054310; Acta Phys. Pol. B34 (2003) 2147.

16. A. Algora, J. Cseh, J. Darai, P. O. Hess, in preparation

17. G. A. Lalazissis and M. M. Sharma, Nucl. Phys. A 586 (1995) 201. 
Copyright of AIP Conference Proceedings is the property of American Institute of Physics. The copyright in an individual article may be maintained by the author in certain cases. Content may not be copied or emailed to multiple sites or posted to a listserv without the copyright holder's express written permission. However, users may print, download, or email articles for individual use. 